\title{
Improved analysis of plasmasphere motion using the VLA radio interferometer
}

\author{
G. W. Hoogeveen, A. R. Jacobson \\ Space and Atmospheric Sciences Group, Mail Stop D466, Los Alamos National Laboratory, Los Alamos, NM 87545 \\ Received: 31 October 1995 / Revised: 8 July 1996 / Accepted: 4 September 1996
}

\begin{abstract}
Observations using the very large array (VLA) radio interferometer during the past five years have enabled the discovery of a new type of plasmasphere disturbance, the magnetic eastward-directed wave. Previous work indicated these disturbances were likely frozen to the geomagnetic field as determined from their azimuth distributions. This work provides a method to explain more accurately the azimuth distribution, thereby allowing the calculation of the disturbances' location in the plasmasphere independently of the measured velocity. The measurable velocity due to corotation is calculated and subtracted from the measured trace velocity. This difference, or deviation from corotation, is attributed to electrodynamic convection; the measurement of plasmaspheric convection may lead to the eventual monitoring of mid-latitude electric fields. Disturbances are seen convecting predominantly westward, with the fastest having angular velocities greater than the anticorotating VLA line of sight. The direction of convection and conditions of observations indicate that the disturbances are likely the same phenomenon seen by the Los Alamos satellite beacon array.
\end{abstract}

\section{Introduction}

The very large array (VLA) radio-interferometer (Thompson et al., 1980), whose primary mission is to carry out radio astronomy, nevertheless is quite capable of measuring line-of-sight transient total electron content (TEC) irregularities. The VLA has 27 independent radio antennas, each of which has the capability of recording multiple frequencies simultaneously. Each antenna measures the electrical phase advance due to the $307.5-\mathrm{MHz}$ signal passing through the plasmasphere/ionosphere system. The phase time-series for

Correspondence to: G. Hoogeveen every antenna is then differenced with a reference antenna resulting in 26 independent baselines. In this way, the VLA can detect baseline differences in TEC to the order of $10^{13}-10^{14} \mathrm{~m}^{-2}$ (Carlos and Massey, 1994). This allows for the examination of very subtle disturbances undetectable by in situ spacecraft or by many ground-based observations. Previous work done with the VLA discovered a new phenomenon of transient disturbances in the plasmasphere termed magnetic eastward-directed disturbances, or MEDs, because their directions of propagation, or azimuths, were clustered around magnetic east at the VLA, $102^{\circ}$ (Jacobson and Erickson, 1992a,b; Jacobson and Erickson, 1993, hereafter referred to as JE93).

Figure 1 shows an idealized sketch of MED event's TEC signature $\left(1\right.$ radian of electrical phase $=3.6 \times 10^{14}$ electrons $\mathrm{m}^{-2}$ of TEC for $307.5-\mathrm{MHz}$ radio waves) as it propagates across the VLA. The lines indicate the phasefronts of the MED wave. Each independent baseline contributes information on the phasefront's motion. By modeling these phase changes as a propagating plane wave, several parameters can be extracted from the data. As shown in Fig. 1, the azimuth is defined to be the direction (reported as an angle measured in degrees clockwise from north) in which the disturbance appears to move. The azimuth is necessarily perpendicular to the phasefronts, and by JE93's simple selection criterion for MEDs, the azimuth was chosen to lie within $\pm 15^{\circ}$ of $102^{\circ}$ (magnetic East at the VLA). Next, the wavelength, or equivalently the wave vector, can be extracted from the data. Finally, a knowledge of the temporal frequency of the passing wave fronts allows a measurement of the trace velocity across the array. The trace azimuth and velocity of the waves are the most important characteristics of MEDs, characteristics which reveal most about their nature.

JE93 concluded that MED events cannot be ionospheric disturbances, for several reasons explained therein. Rather, they concluded that MED disturbances are an effect of an instability in the plasmasphere causing electron-density enhancements elongated along 


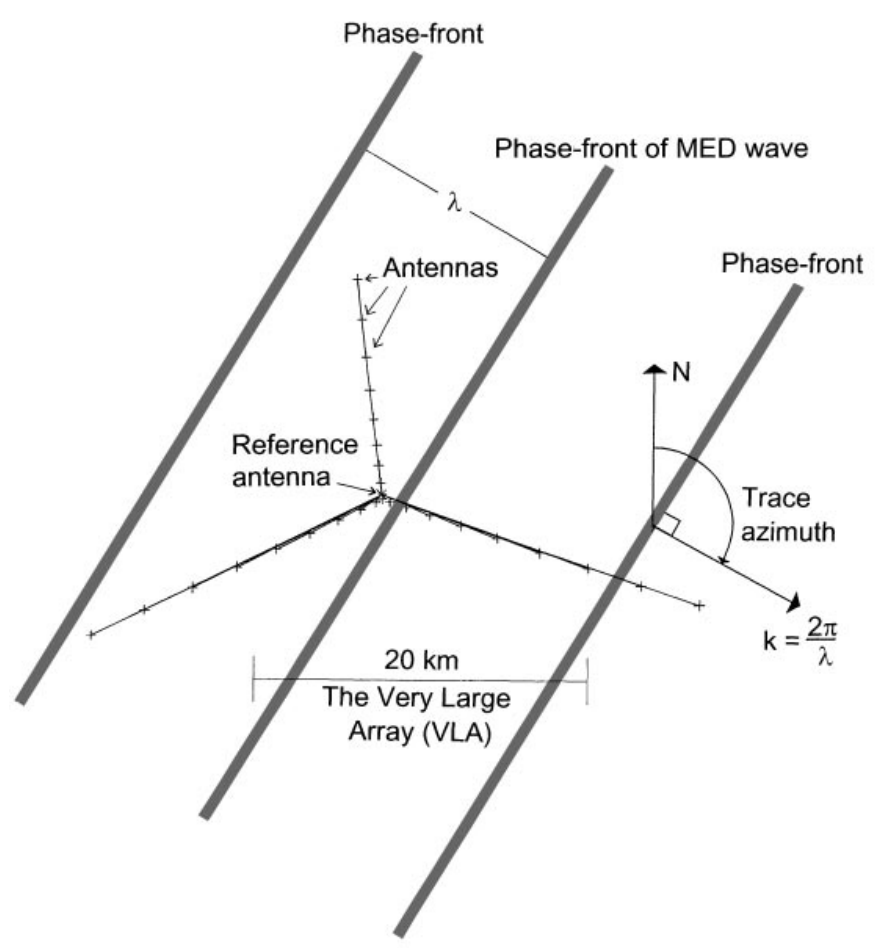

Fig. 1. Idealized sketch of the VLA and a MED wave passing across the array. The trace azimuth is shown as the angle clockwise from north, and the wave vector, $\boldsymbol{k}$, is defined to be equal to $2 \pi / \lambda$ in the direction of the trace azimuth. Note the spatial scale

magnetic field lines. Recently, it has been postulated that the instability responsible for these density structures could be the interchange instability on density gradients in the plasmasphere (Jacobson et al., 1996). It is the field-aligned nature that causes the events' azimuths to cluster around magnetic eastward as the VLA's line of sight slips westward. Although the previous study was generally correct in its conclusions, it made two incorrect assumptions that caused some inaccurate results.

The incorrect assumptions in the JE93 study were (1) the MED's trace azimuth was the in situ zonal direction ( $+\hat{a}$ in Fig. 2) projected onto the VLA plane (the tangent to the spherical surface of the Earth at the VLA), and (2) this $\hat{a}$-projected direction should be $102^{\circ}$ clockwise from north at all times. Figures 3 and 4 show that these two assumptions are incorrect. Figure 3 displays a histogram of the measured trace azimuth distribution of all planewave events (top) and the difference between their azimuths and the instantaneous projected $\hat{a}$ direction (for each individual event) on the VLA plane (bottom). If the azimuths were aligned (or polarized) along $\hat{a}$ in the plasmasphere, there should be a large cluster of events at $0^{\circ}$ in the bottom panel of Fig. 3. As is seen, there is no better clustering at $0^{\circ}$ than the original clustering around $102^{\circ}$ in the top; the $\hat{a}$-polarization model does not explain the azimuth distribution. Figure 4 demonstrates how the in situ zonal direction $(+\hat{a})$ at $L=2.2$ (a representative location for all MEDs, as can be seen later in Fig. 9) projected onto the VLA plane changes

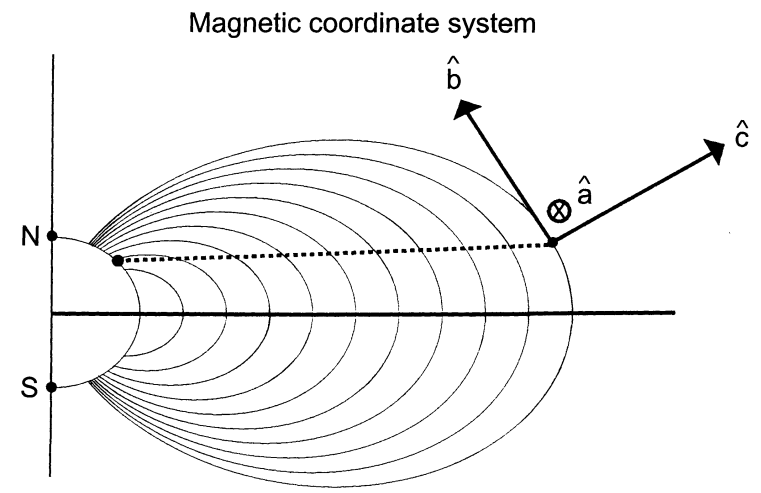

Fig. 2. Magnetic coordinate system used to identify in situ directions: $\hat{b}$ is a unit vector in the magnetic-field direction; $\hat{a}$ is a unit vector in the magnetic zonal direction; and $\hat{c}$ is a unit vector in the magnetic meridian, orthogonal to $\hat{a}$ and $\hat{b}$

with the westward component of the line of sight (see Fig. 5 for the definition of the angle $\xi$ ). For every planewave event modeled (in the JE93 event pool), we projected the $+\hat{a}$ direction from $L=2.2$ along that instantaneous line of sight onto the VLA plane, the
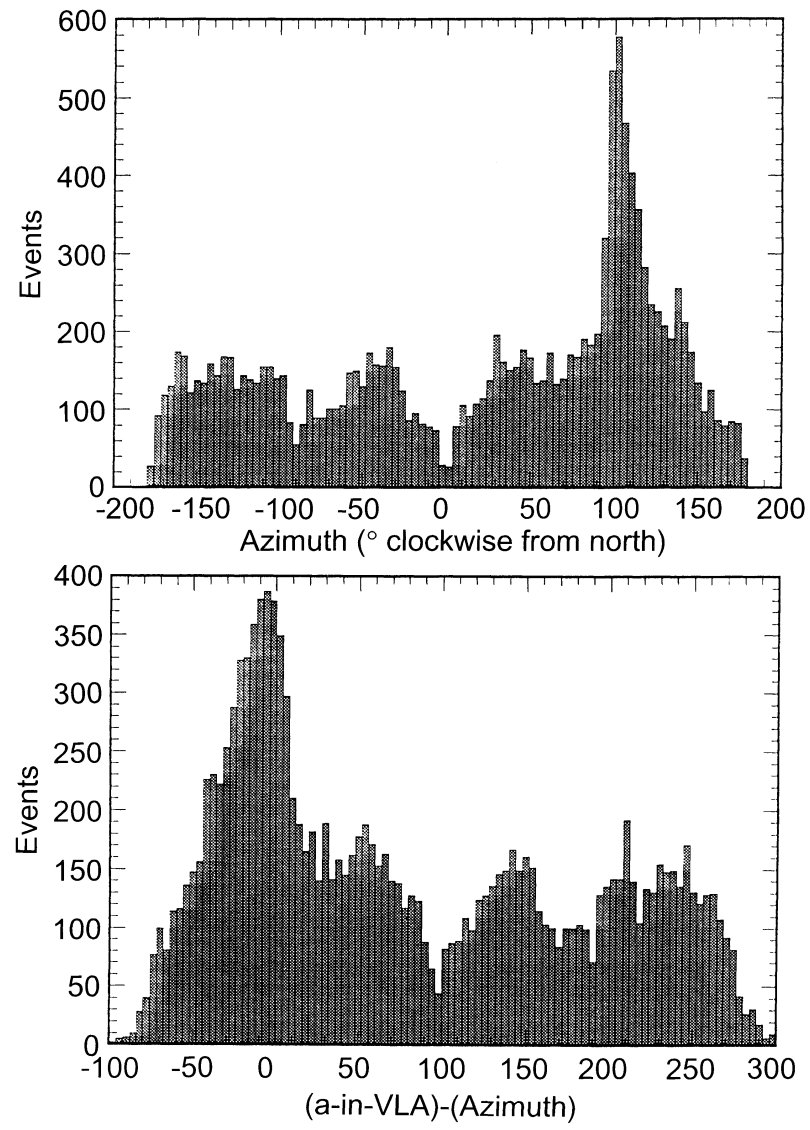

Fig. 3. (Top) Histogram of measured trace azimuths. Azimuths are measured clockwise from geographic north. There are 100 bins, each $4^{\circ}$ wide. (Bottom) Histogram of $\hat{a}$-projected-onto-the-VLA-plane minus azimuth. If the VLA measured $\hat{a}$-projected, this histogram would be clustered around $0^{\circ}$ and $180^{\circ}$ (for MED and retrograde events, respectively) 


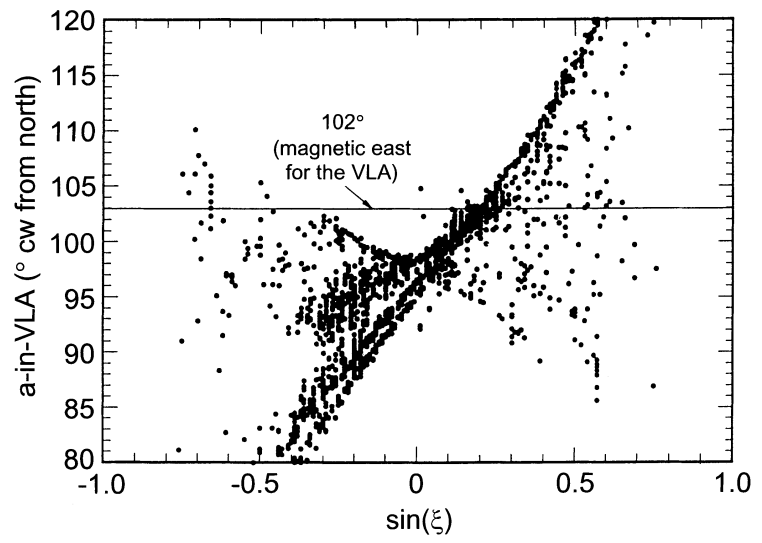

Fig. 4. Relationship between $\sin (\xi)$ (see Fig. 5) and $\hat{a}$-projected-ontoVLA-plane. For every event in the JE93 event pool, we project the $\hat{a}$ direction at $L=2.2$ along the line of sight (see text for the reason behind this number) onto the plane of the VLA. As the line of sight moves westward, $\hat{a}$-projected moves clockwise; it is equal to $102^{\circ}$ for only a limited range of look directions just north of the meridian

results of which are graphed in Fig. 4. The assumption that $\hat{a}$-in-VLA is always $102^{\circ}$ is obviously a poor one, since very few dots fall on the line.

These two simplistic assumptions led to a less-thanfull exploitation of the full information provided by the azimuth data. First, recall that MEDs were identified in JE93 as those events possessing trace azimuths within $\pm 15^{\circ}$ centered on magnetic east at the VLA $\left(102^{\circ}\right)$. This MED definition ignored the details of how appropriately projected magnetic east changed as the line of sight moved from east to west. The projection of $\boldsymbol{B}$, and hence the projected magnetic-east direction, was not calculated for every $\xi$, as it was for Fig. 4. Therefore, JE93 threw out many MED events due to the imposition of overly restrictive selection criteria. Second, MEDs (in JE93) were assumed to move at $102^{\circ}$, and were assumed to be polarized in the $+\hat{a}$ direction (i.e., $\hat{a}$ was assumed to be the direction from one density enhancement to its nearest neighbor). JE93 then projected $+\hat{a}$ onto the VLA plane (for each event) and assumed that the measured trace velocity had to match the corotation velocity in this $\hat{a}$-projected-onto-VLA direction. The resultant $L$-shells calculated by equating these velocities are incorrect because the motion of the phase fronts in the VLA plane are in no way correlated to the polarization of the in situ disturbances, as will be shown.

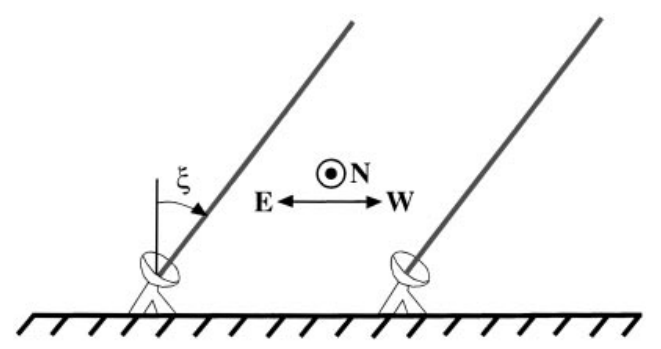

Fig. 5. Schematic view from the north of two elements of the array. The sine of the angle, $\xi$, is the westward component of the line of sight
This study introduces the simple concept of the shadows of the magnetic field projected onto the VLA, resulting in important differences from the JE93 study. If MEDs are elongated along the magnetic field, then their TEC signatures projected onto the array must appear to be the shadows of the field lines themselves. Further, the MEDs' azimuth must be perpendicular to these shadows of the magnetic field, since the shadows appear infinite in length at the array. Finally, by matching the azimuth of an event with the perpendicular field shadow we are able to locate the events along the line of sight in the plasmasphere. These realizations allow for improved, and more inclusive, identification of MED events, as well as a reliable method of placing events in the plasmasphere independently of the trace velocity. These new results enable measurements of plasmasphere macroscopic motion, and could eventually enable realtime monitoring of mid-latitude plasmaspheric electric fields.

\section{Previous study}

The previous study of VLA-detected MED disturbances (JE93) analyzed 343 source hours of data. The data was least-squares fitted (with input from all independent baselines) to a plane wave

$N(\omega)=A(\omega) \exp \{-i \boldsymbol{k} \cdot \boldsymbol{r}\}$

where $N(\omega)$ is the Fourier-transformed TEC time-series, $A(\omega)$ is the amplitude, $\boldsymbol{k}$ is the wavevector, and $\boldsymbol{r}$ is the horizontal position of the antennas. Out of a possible $2.4 \times 10^{5}$ parameter solutions, roughly $7 \%$ were well described by a singular plane wave propagating across the array. These plane waves were then defined to be an event centered in time on that window. To be more explicit, an event is a monochromatic wave whose Fourier frequency was above $4 \mathrm{mHz}$. Of these planewave events, it was noticed that most all those with trace velocities greater than $200 \mathrm{~m} / \mathrm{s}$ possessed azimuths of $102^{\circ} \pm 15^{\circ}$; a small set had azimuths directly $180^{\circ}$ opposite this and were labelled "retrograde" events. Finally, by comparing the modeled velocities with corotation, the $L$-shells of the events were found; they were distributed from $L=1.6$ to 4.0 , with a maximum near 2.2, and a small tail out to larger $L$ values.

Although the same $L$-shell nature will prove to be correct, the events identified as MED, as well as the velocity distributions of MED events, will be shown to be better determined in the present study.

\section{New data}

Before describing the revised method of identifying MEDs and their location in the plasmasphere, data obtained since the JE93 study shall be discussed. Our database has been increased by 51 source hours. The database now contains approximately 394 source hours, a $15 \%$ increase in observations from JE93. The interested reader is referred to the previous papers 
(Jacobson and Erickson, 1992a,b; JE93) for a thorough explanation of the data reduction scheme. In the end, the time-series TEC data is transformed into some number of plane-wave solutions, each described by a trace velocity and azimuth. Again, the total number of acceptable plane-wave solutions is roughly $7 \%$ of the total number of possible observations.

In order to eliminate some noise contamination, an analysis of the radio-frequency (rf) noise was made. The VLA observes a (presumed) single cosmic radio source in two frequencies, 307.5 and $333 \mathrm{MHz}$, simultaneously. Any differences in the measured phase between these two frequencies (excluding the expected plasma scaling $\sim f^{-1}$ ) can be attributed to external rf noise. For the present study, we reanalyzed all 394 hours of data and found the average noise value (by differencing the two rf frequencies' scaled data) for each Fourier frequency of the fitted data, shown in Fig. 6. We then compared each solution value of the fitted Fourier frequencies, and if the signal did not exceed twice the noise value for that frequency, that solution was eliminated.

It is quite obvious from Fig. 6 that the 1991, 1992, and 1993 data (dashed line) are more severely affected by rf noise and source confusion than are the 1990 and 1994 data (solid line). This is a result of the fewer baselines employed for those campaigns; the mitigation of source confusion is directly proportional to the number of the baselines in the array (Jacobson and Erickson, 1992a). This rf-noise study not only is useful for elimination of bad plane-wave solutions, but also gives us an idea of the sensitivity of the instrument. It was previously stated that the sensitivity was of the order of $10^{13}$ to $10^{14} \mathrm{~m}^{-2}$. With the use of Fig. 6 we see that the value is frequency dependent, and the integrated noise from 4 to $20 \mathrm{mHz}$ (the passband used) is $1.9 \times 10^{13} \mathrm{~m}^{-2}(0.050$ radians $)$ for the fewer antenna (usually 9) sessions during 1991-1993, and $1.4 \times 10^{13} \mathrm{~m}^{-2}(0.038$ radians $)$ for the complete 27 -

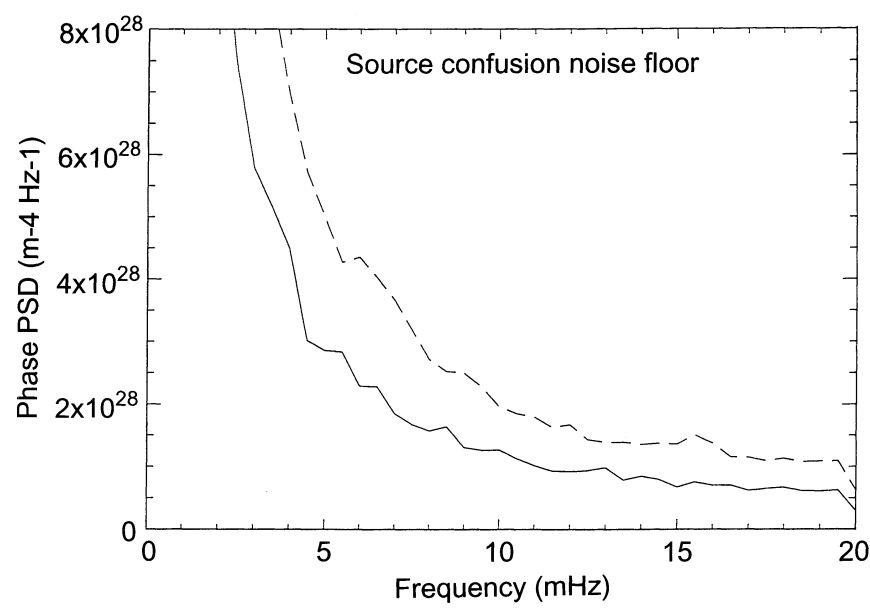

Fig. 6. Radio-frequency noise spectrum calculated from the difference of two simultaneously measured frequencies, 307.5 and 333 MHz. The dashed line represents data taken during the 1991-1993 sessions (fewer antennas per array) and the solid line represents data from sessions during 1990 and 1994 (all available antennas in one array) antenna sessions during the 1990 and 1994 sessions.

Implementing this noise reduction scheme results in 14000 valid events, compared to 16900 events from JE93, even though the database has been expanded by $15 \%$. Therefore we have eliminated roughly $40 \%$ of the highest noise- (or confusion-) contaminated events, leaving a cleaner, more noise-free database of actual TEC disturbances.

\section{New interpretation - magnetic-field shadows}

This study proposes that the shadow of the in situ field line projected onto the VLA plane, and not the in situ polarization, is the relevant parameter when modeling MEDs. The argument supporting this statement is best given through the following very simple gedanken experiment.

Imagine a flux tube in the inner plasmasphere filled with plasma (i.e., the MED electron-density enhancement). Imagine several flux tubes spaced evenly in the magnetic zonal direction ( $\pm \hat{a}$ in Fig. 2 ) on the same $L$ shell. Now imagine a flashlight (flash-radio, if you will) illuminating these flux tubes from infinity (unresolved radio source) and casting their shadows onto the spherical surface of the earth at the VLA. Assume the array is small compared to the long dimension of the flux-tube shadows such that from this point the shadows appear infinite in length. These flux-tube shadows look like lines with a similar appearance to the phase fronts shown in Fig. 1. Next, imagine the Earth and the flux tubes (assuming for simplicity they are corotating) rotating as the flashlight stays fixed in space. The shadows on the VLA now move in the opposite direction to the rotation of the Earth, and perpendicular to the shadows themselves. If the VLA motion due to the Earth's rotation was along the length of the sausage shadows, no motion would be apparent. Further, since the magnetic axis is not aligned with the rotational axis the flux-tube motion will not be precisely perpendicular to the shadows. Now back up a couple of steps, and imagine the flux tubes are spaced evenly in the meridional direction ( $\hat{c}$ in Fig. 2), instead of in the $\hat{a}$ direction. If the flashlight then illuminated the links from anywhere but the local meridian, the observed shadow movements due to the rotation of the Earth would be indistinguishable from the $\hat{a}$-aligned case.

Two important points can be gained from this simple gedanken experiment. First, the phase fronts are merely the shadows of the magnetic field projected onto the VLA plane, and furthermore, can only move in the direction perpendicular to themselves (i.e., motion parallel to the shadows is unobservable). Second, the in situ polarization is irrelevant to the observable motion of the phasefronts; the VLA only observes motion of the $2 \mathrm{D}$ projection of electron flux tubes, no matter what their 3D spacing may be.

This new interpretation of the in-array motion is easily tested. If the phase fronts are, in fact, the shadows of magnetic field lines, then the histogram of the difference between the azimuth of an event and the 
shadow of its field line should be sharply clustered around $\pm 90^{\circ}$ (for magnetic eastward and westward events, respectively). It is worth mentioning again that the shadow is calculated separately for each event using the representative $L=2.2$ along its line of sight. Figure 7 shows the difference between the $\boldsymbol{B}$-shadow direction and the azimuth. It is quite apparent that the $\boldsymbol{B}$-shadow model more successfully explains the azimuth distribution than the $\hat{a}$-aligned model shown in Fig. 3. Furthermore, both the magnetic eastward $\left(+90^{\circ}\right)$ and westward $\left(-90^{\circ}\right)$ events are evident; the magnetic westward events, formerly known as retrograde events in the JE93 study, are not easily identified in the data histogram of Fig. 3 (top), nor in the $\hat{a}$-aligned model histogram of Fig. 3 (bottom). Also, notice the roughly 100 events/bin isotropic background of apparently nonMED events. With 100 bins, there are approximately $10^{4}$ non-MED events in this data set of rf-noise-free plane waves.

The $\boldsymbol{B}$-shadow interpretation allows for a reliable method of placing the disturbances along the line of sight. Figure 8 shows the direction of $\boldsymbol{B}$ shadow on the VLA plane along three representative lines of sight at distances from $L=2.0$ to 3.5 . Notice in each instance the change in $\boldsymbol{B}$ shadow is monotonic with distance along the line of sight. Furthermore, some lines of sight have a greater overall change in $\boldsymbol{B}$ shadow from $L=2$ to 3. The VLA happened to be looking along these "sensitive" lines of sight, defined as

$\Delta B_{s h} \equiv\left|B_{s h}(L=3)-B_{s h}(L=2)\right| \geq 20^{\circ}$

where $B_{s h}$ is the direction of the shadow on the array clockwise from north approximately $40 \%$ of the time. Therefore, roughly $40 \%$ of the number of MED events have lines of sight which allow firstly the accurate placement of disturbances in the plasmasphere and secondly the calculation of in situ convection.

The placement of MEDs in the plasmasphere is accomplished through a simple algorithm. The algorithm consists of moving out along the line of sight for

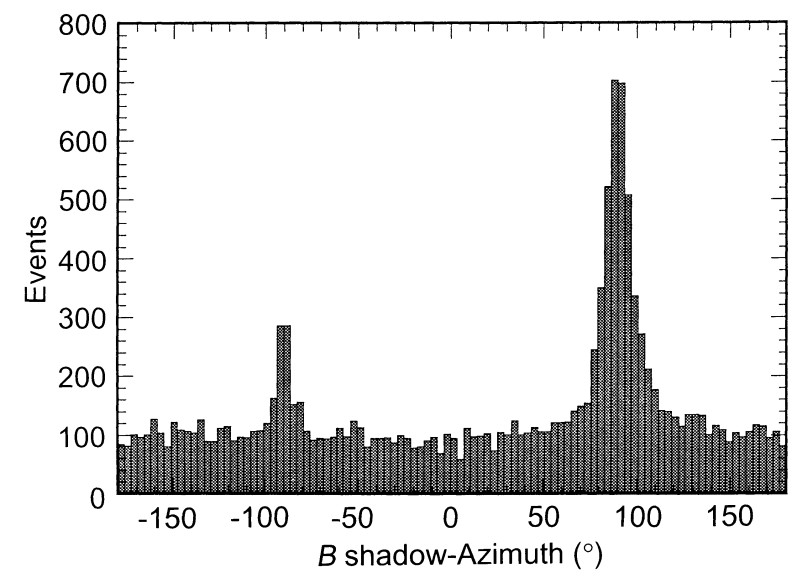

Fig. 7. Histogram of the difference between $\boldsymbol{B}$ shadow and trace azimuth. Notice the sharp peaks at azimuths $\pm 90^{\circ}$ from $\boldsymbol{B}$ shadow, representing the MED and retrograde events, respectively

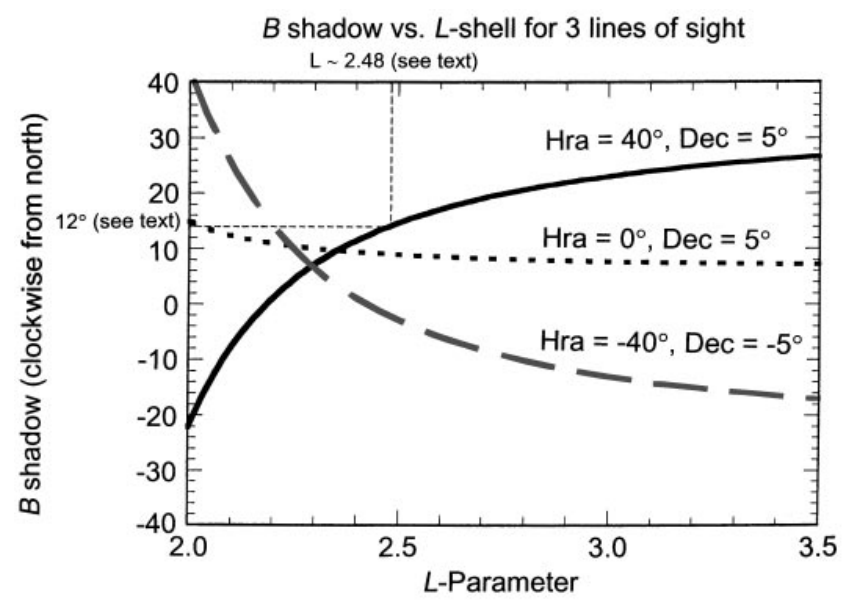

Fig. 8. Direction of $\boldsymbol{B}$ shadow (in degrees clockwise from north) as a function of the McIlwain parameter $(L)$ for three different lines of sight. The hour angles and declinations for each line of sight appear next to each curve. The corresponding $L$ parameter for a $\boldsymbol{B}$ shadow of $12^{\circ}$ is approximately 2.48 for a line of sight with hour angle $=40^{\circ}$ and declination $=5^{\circ}$ (see text)

each event, projecting the $\boldsymbol{B}$ shadow onto the VLA at various radii, and comparing it to the event's trace azimuth. If the $\boldsymbol{B}$ shadow projected from a given distance along the line of sight is perpendicular to that event's trace azimuth, then the position is found. This position is then converted to the McIlwain parameter, $L$ (Mc Ilwain, 1961). Figure 9 shows the $L$-shell distribution of the MED data. A close comparison of Fig. 9 to JE93's result reveals that the present study places MEDs on smaller $L$-shells than JE93. The inclusion of slower events by eliminating one JE93 MED criterion $\left(V_{\text {trace }}>150 \mathrm{~m} / \mathrm{s}\right)$ results in a skewing of the $L$-shell distribution to smaller values. Incidentally, the low number of events (1608) in Fig. 9 is a result of using only the "sensitive" lines of sight (40\% of the total). Regardless, the conclusion that MED disturbances are inner-plasmasphere phenomena remains; the MEDs we

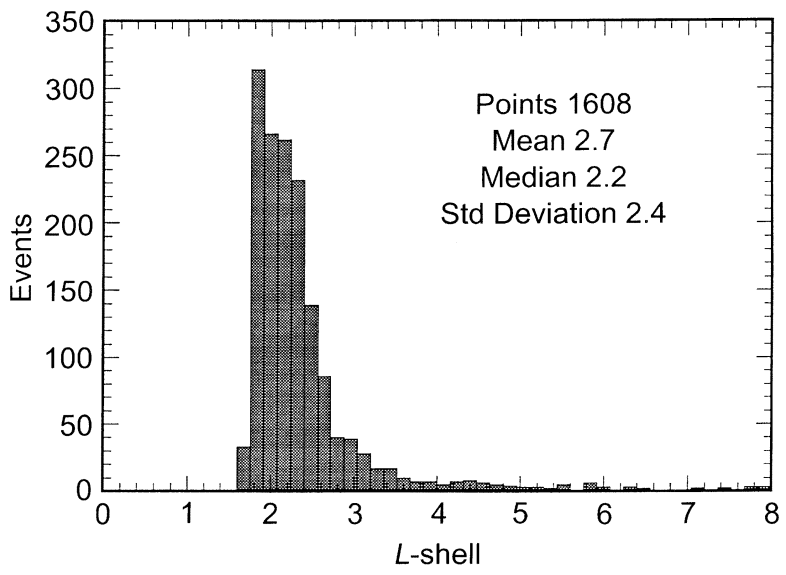

Fig. 9. Histogram of inferred $L$-shell for MED events with lines of sight for which distance placement was possible (see text and Fig. 8). The distribution is skewed to smaller values in comparison to JE93 results. However, this figure shows MEDs are inner-plasmasphere phenomena, thus agreeing with the previous study's conclusion 
observe predominantly exist from $L=1.6$ to 3.0 , with a small, and statistically insignificant tail out to 8 .

This $L$-shell range, 1.6-3.0 is partly an artifice of the observation geometry, as well as the nature of TEC measurements. First, the VLA has a geomagnetic latitude of approximately $42^{\circ}$ north which corresponds to $L=1.8$ (assuming altitude $=0$ ), and the magnetic field has a dip angle of $11^{\circ}$. Therefore, it is only possible to measure $L$-shells smaller than 1.8 when looking in the extreme southern sky. There simply weren't many lines of sight with elevations less than $11^{\circ}$, resulting in an inability to measure plasmaspheric disturbances on $L$ shells much less than 1.8. Second, TEC measurements are dominated by ionospheric plasma. The disturbances discussed in this study are less $1 \%$ of the background TEC. Therefore, in order for the TEC disturbances to lie above our instrument sensitivity they had to have large changes in TEC, biasing our measurements to higher plasma-density regions, or lower $L$-shells. This effect contributes to the fall-off in events outside $L=3$ in Fig. 9.

The algorithm described previously for placing MEDs in the plasmasphere also eliminates the $\sim 10^{4}$ event background seen in Fig. 7. A MED was formerly (JE93) identified if $f_{\text {wave }}>4 \mathrm{mHz}, 87^{\circ} \leq$ azimuth $\leq 117^{\circ}$, and $V_{\text {trace }}>150 \mathrm{~m} / \mathrm{s}$. We now identify an MED as an event whose trace azimuth lies perpendicular to a $\boldsymbol{B}$ shadow projected from a range of $L=1.6$ 8.0 (see Fig. 9) along the line of sight, as well as retaining the $f_{\text {wave }}>4 \mathrm{mHz}$ criterion; no $V_{\text {trace }}$ criterion is imposed. The trace azimuth histogram for this newly identified set of MEDs is shown in Fig. 10. By comparing Figs. 10 and 7 it appears that the nonMED background is gone. In fact, the number of events in Fig. 7 is 14017 and the number of newly defined MEDs in Fig. 10 is 4581; the difference is roughly $10^{4}$.

The second ability provided by the $\boldsymbol{B}$-shadow interpretation may prove to be the most powerful. Since locating a MED in the plasmasphere is now done independently of the trace velocity of the wave, a comparison of the trace velocity and the in situ corotation velocity projected along the trace azimuth

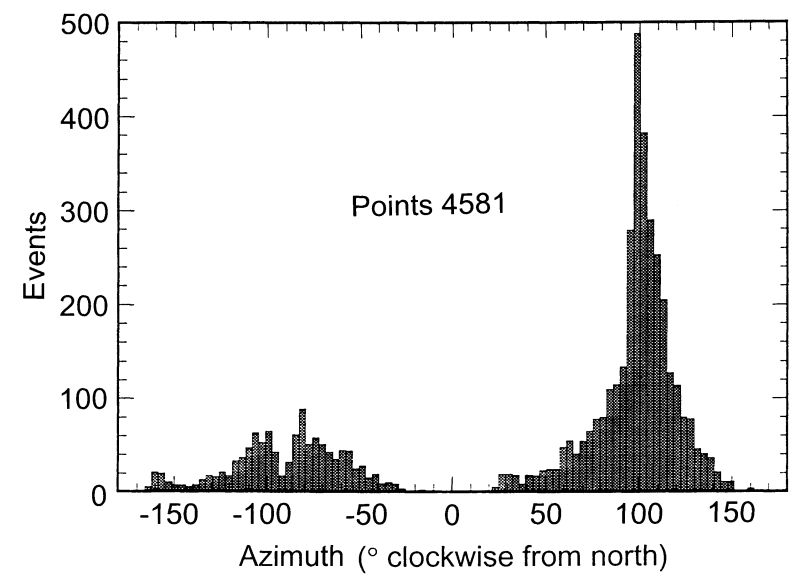

Fig. 10. Histograms of trace azimuth for the newly defined MEDs. The background events seen in Fig. 7 have been removed reveals information about the convection of the plasmasphere. For example, assume a disturbance propagates across the VLA with a trace azimuth of $102^{\circ}$ and a trace velocity of $400 \mathrm{~m} / \mathrm{s}$, for a line of sight with an hour-angle of $40^{\circ}$ and a declination of $5^{\circ}$. Then, Fig. 8 shows that this wave moving with an azimuth of $102^{\circ}$ (i.e., $\boldsymbol{B}$ shadow of $102^{\circ}-90^{\circ}=12^{\circ}$ ) must originate from a disturbance at $L \approx 2.48$. The location $L \approx 2.48$ along the given line of sight is converted into altitude, longitude, and latitude and the resultant corotation velocity is calculated. For illustration, let us assume the corotation velocity is $570 \mathrm{~m} / \mathrm{s}$. However, in order to compare these two velocities, they must first both be projected onto the plane perpendicular to the line of sight. Assume that the properly projected trace velocity thus changes from 400 to $350 \mathrm{~m} / \mathrm{s}$. Furthermore, assume the component of the corotation velocity projected onto this plane is $475 \mathrm{~m} / \mathrm{s}$, and then when projected onto the direction of the trace velocity in this plane, becomes $450 \mathrm{~m} / \mathrm{s}$. The difference between the appropriately transformed trace velocity and corotation velocity is $-100 \mathrm{~m} / \mathrm{s}$, i.e., $100 \mathrm{~m} / \mathrm{s}$ in the direction $180^{\circ}$ from the trace azimuth, or $282^{\circ}$. The apparent magnetic eastward-directed wave has thus become an in situ westward-convecting disturbance with a projected convection velocity onto the plane perpendicular to the line of sight, and perpendicular to the magnetic-field direction in this plane, of $100 \mathrm{~m} / \mathrm{s}$.

The complete results of this analysis are reported in Fig. 11, which demonstrates, firstly, more events were found to convect westward, and secondly, westward events moved faster. The median eastward-convection velocity (component perpendicular to both $\boldsymbol{B}$ and the line of sight) was $70 \mathrm{~m} / \mathrm{s}$, taken from a total of 367 events, and the median westward-convection velocity was $170 \mathrm{~m} / \mathrm{s}$ from 1236 events. It is important to remember that this convection analysis was performed on only those events whose lines of sight allowed a high

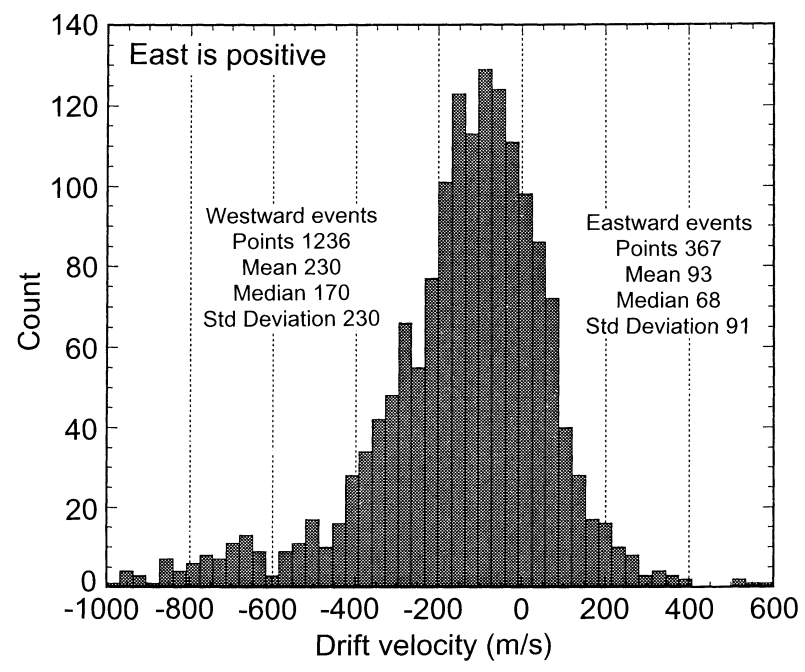

Fig. 11. Drift-velocity magnitudes for westward and eastward events. Eastward events are taken to be positive and westward events are negative. The more numerous westward-drifting events travel significantly faster than the eastward events 
level of precision in placing the events in the plasmasphere (hence the small counts). These events were identified by accepting only the lines of sight which had more than $20^{\circ}$ differences in $\boldsymbol{B}$ shadow at $L=2.0$ and 3.0 (Eq. 1). This ensured accurate calculations of the corotation velocities involved in the convection analysis.

The first result just mentioned brings into question the meaning of MED. Originally, MED described a unique set of waves propagating towards the magnetic eastward direction. However, with the new identification of the events, (i.e., those perpendicular to $\boldsymbol{B}$ shadow) and the subsequent calculation of plasmaspheric convection, the majority of MEDs are now identified as in situ westward-propagating disturbances. The original observations indicated an eastward-propagating phenomenon; however, the new interpretations reveal a westward-propagating plasmaspheric phenomenon. Nevertheless, the causative agents for these waves, the in situ plasma-density structures, are still unique, and previous to JE93, undiscovered phenomenon. Therefore, the unique phenomenon termed MED no longer simply refers to a magnetic eastward-directed wave, rather, it refers to the density structure whose shadows on the Earth appear to move eastward when illuminated from a fixed point in space, even though in the frame of the corotating plasmasphere the structures move westward.

In any case, the convection velocities, or the deviation-from-corotation velocities, are the components of the in situ convection velocities perpendicular to the line of sight. These velocities, if assumed to be in the $\pm \hat{a}$-direction, are directly proportional to the cylindrical radius from the rotational axis of the planet. Figure 12 plots the relative magnitude of $\hat{a}$ projected onto the plane perpendicular to the line of sight (see fig. caption). It is quite obvious that our lines of sight (in this data set) are much more sensitive to $\hat{a}$ motion than than $\hat{c}$ motion, and therefore we assume our events move in the $\pm \hat{a}$ direction. A better method of comparing zonal drift speeds is to map them down to

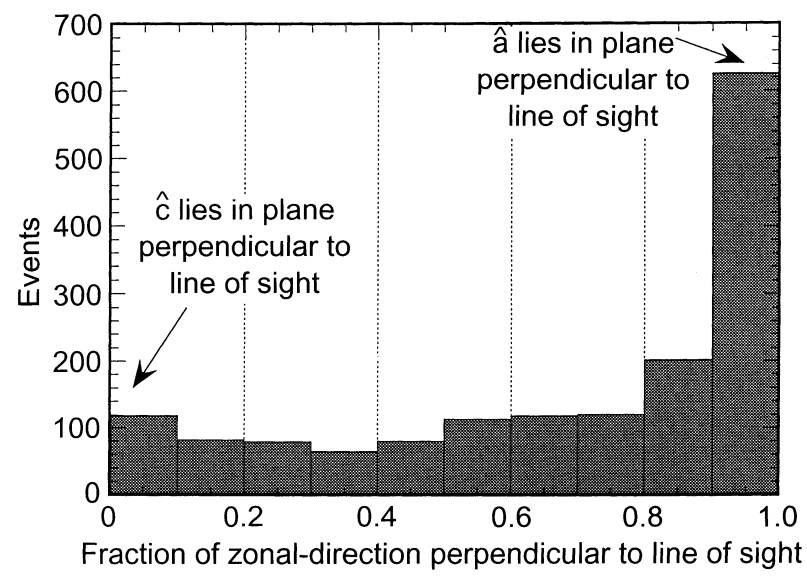

Fig. 12. The ratio of $\hat{a}$-perpendicular to the line of sight to the sum of $\hat{a}$-perpendicular and $\hat{c}$-perpendicular to the line of sight. This is a measure of the sensitivity of the instrument, for a particular line of sight, to drifts in the zonal, $\hat{a}$, direction a standard height. Since velocities in the F-layer are well known (e.g., Mazaudier and Venkateswaran, 1990; Fejer et al., 1981; Kudeki et al., 1981), we map all drift velocities to $300 \mathrm{~km}$. Given the equation of a dipole field line

$R \equiv \frac{r}{R_{E}}=L \cos ^{2}\left(\lambda_{m}\right)$

where $\lambda_{m}$ is the magnetic latitude, and as stated above

$\frac{V_{f}}{V_{i s}}=\frac{r_{f}}{r_{i s}}$,

where $r_{f}$ denotes the cylindrical radius to the F-layer, and $r_{i s}$ denotes the in situ cylindrical radius, the velocity in the F-layer can be written as

$V_{f}=V_{i s}\left(\frac{1+\frac{300 \mathrm{~km}}{R_{E}}}{1+\frac{\mathrm{alt} \mathrm{tis}_{E}}{R_{E}}}\right)^{\frac{3}{2}}$,

where alt $_{i s}$ is the altitude of the in situ disturbance. This mapping scheme produced the results shown in Fig. 13. The westward-convecting-event histogram remains skewed towards higher speeds than the eastward event histogram. The median F-layer-mapped speed for the westward events is approximately $80 \mathrm{~m} / \mathrm{s}$, while the eastwards-events speed is $40 \mathrm{~m} / \mathrm{s}$. The inferred F-layer drift-speed magnitudes are in broad agreement with those seen at Millstone Hill (Jacobson et al., 1996). Drift-speed comparisons are made with Millstone Hill since our array lies nearest in magnetic latitude to it than to any other mid-latitude observatory (VLA-42 ${ }^{\circ}$; Millstone- $56^{\circ}$ ).

\section{Retrograde Events}

Another benefit of the newly identified set of MED events, is the inclusion (and proper interpretation) of many more, what JE93 termed, "retrograde" events. JE93 reported 240 events with azimuths within $\pm 15^{\circ}$ of $-68^{\circ}$, predominantly seen in northern skies (angle north

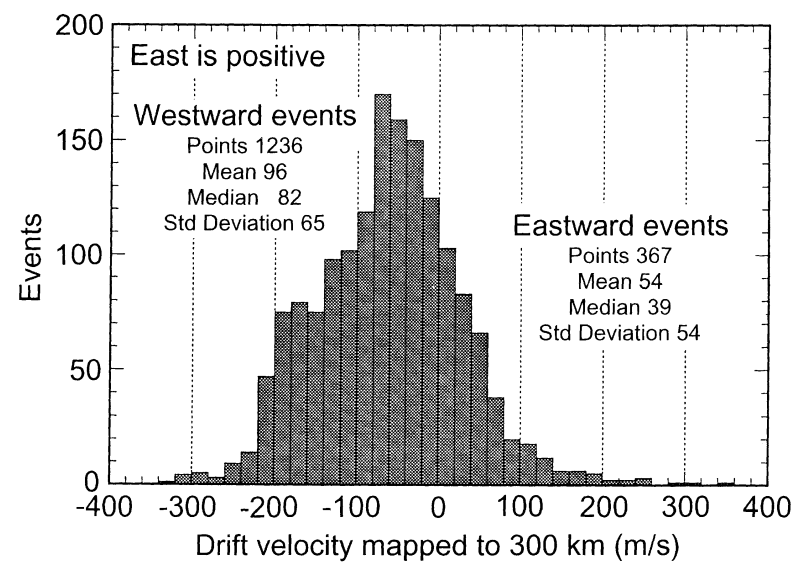

Fig. 13. Results of mapping each individual event in Fig. 11 down, along the field line, to an altitude of $300 \mathrm{~km}$ (F-layer). Notice that the westward events (negative values) still travel with a higher speed than the eastward events (positive values) 

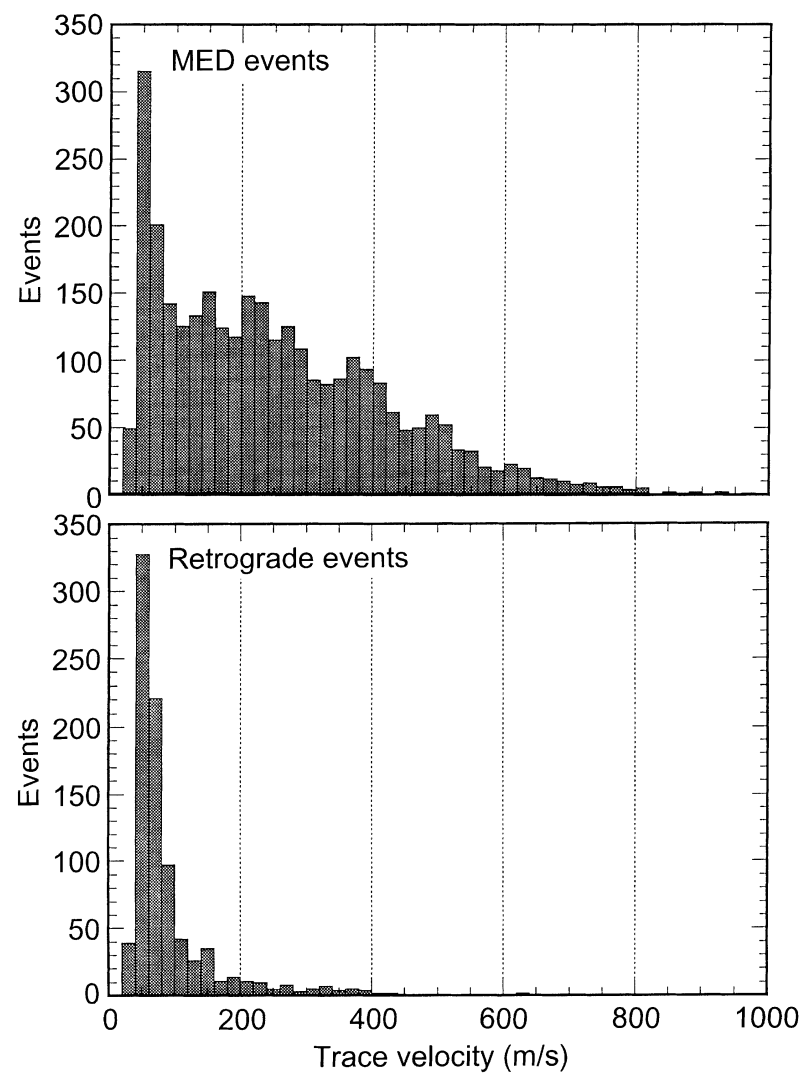

Fig. 14. Histograms of (top) MED events measured trace speed and (bottom) retrograde events ( $+90^{\circ}$ to $\boldsymbol{B}$ shadow) measured trace speed. It is most obvious that the retrograde events move more slowly across the VLA plane

from zenith $>0^{\circ}$ ), disturbed magnetic times (high $K_{p}^{\prime}$ ), and evening hours. They labeled these events "retrograde" due to their apparent anticorotational azimuth, and tentatively interpreted them as "peeled off outerregions of the plasmasphere," due to their affinity for high- $K_{p}$ (Horwitz et al., 1990; Chappell, 1974). This study identifies 886 retrograde events, i.e., events in Fig. 10 possessing azimuths less than $0^{\circ}$. Why are there so many more events in this noise-reduced event pool? The new MED-identification criterion (including both MED and retrograde events) relaxes the JE93 velocity constraint, $V_{\text {trace }}>200 \mathrm{~m} / \mathrm{s}$, thereby identifying many more events. Figure 14 shows the trace velocity distribution of both the MED and retrograde events. It is obvious if we had retained the $200-\mathrm{m} / \mathrm{s}$ criterion for retrograde events, the majority of our events would be lost (only 73 would remain). Furthermore, Fig. 14 demonstrates a very important comparison between retrograde and MED events: retrograde events move more slowly across the VLA plane.

We hypothesize that the retrograde events are in situ disturbances drifting westward faster than the westward-moving line of sight. This means that the in situ velocity of the retrograde events is greater than its corotation velocity, and in the opposite direction, for lines of sight perpendicular to $\hat{a}$, and progressively smaller as the lines of sight become more perpendicular to $\hat{c}$ (since the lines of sight move in the $-\hat{a}$ direction). Assuming a normal convection-velocity distribution, the angular velocity of the abundant, slowly convecting westward events will be greater than the westwardslipping line of sight angular velocity only for lines of sight nearly perpendicular to $\hat{c}$. The top of Fig. 15 indicates that, indeed, retrograde events tend to be seen when the line of sight is perpendicular to $\hat{c}$.

If our hypothesis concerning retrograde events is correct, they should also be seen more often for lines of sight looking in the northern sky. As mentioned in JE93, northern lines of sight imply smaller cylindrical radii, therefore smaller corotation velocities, and therefore the angular velocity of a westward-convecting disturbance could more easily exceed the angular velocity of the westward-slipping line of sight. The bottom of Fig. 15 validates this test; retrograde events are more likely seen on northern lines of sight.

However, both panels in Fig. 15 show that a few retrograde events exist for lines of sight perpendicular to

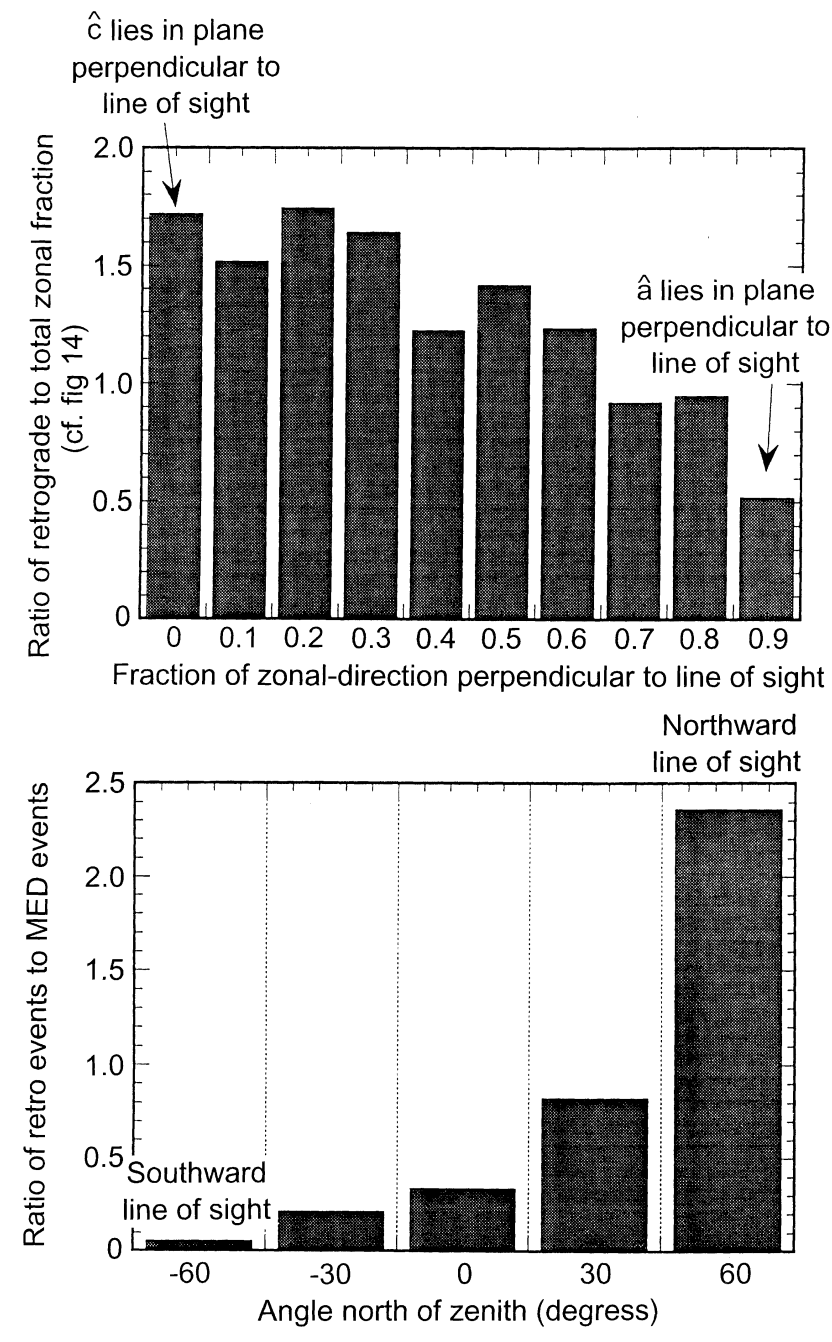

Fig. 15. (Top) Histogram showing that retrograde events are seen along lines of sight relatively insensitive to zonal motion (cf. Fig. 12). (Bottom) Histogram indicating that retrograde events are seen along lines of sight north of zenith 
$\hat{a}$, and in the southern sky, albeit in smaller numbers; there are 116 retrograde events fitting these two criteria. What is the explanation for these events? According to the hypothesis, these events must have extremely large westward-convection speeds in order to exceed the angular velocity of the VLA's line of sight. Figure 16 displays the F-layer drift velocities for these 116 events. Comparing this histogram to the top of Fig. 13, it is obvious that this subset comprises the high-end velocity tail of the westward-drifting population. Finally, Fig. 17 shows that the fast-drifting events preferentially occur during highly disturbed magnetic periods, agreeing with known plasmaspheric behavior (Balmforth et al., 1994; Saxton and Smith, 1991).

How do these findings compare to the JE93 study? Recall that JE93 was unable to identify the majority of this study's retrograde events due to their imposition of the trace-velocity criterion, $V_{\text {trace }}>200 \mathrm{~m} / \mathrm{s}$. Therefore, the only method of comparison between the two studies is to look at the high-trace-velocity retrograde events in this study. Our findings for the 116 fastest-moving retrograde events agree with JE93's conclusion that retrograde events are seen during periods of high $K_{p}$. However, the JE93 interpretation of subrotating plasmasphere patches is no longer necessary. This study reinterprets retrograde events as having one, or both, of the following properties: (1) lines of sight for which $\hat{a}$ motion is not easily measured (i.e., lines of sight perpendicular to $\hat{c}$ ), and/or (2) extremely high westward-convection speeds. In any case, we conclude retrograde events convect westward faster than their westward-moving lines of sight, a fundamentally different conclusion than JE93's subrotating plasmaspherepatch explanation.

\section{Limitations}

The analysis is not completely without weaknesses, and its limitations should be fully realized before any conclusions are reached. For instance, the method by

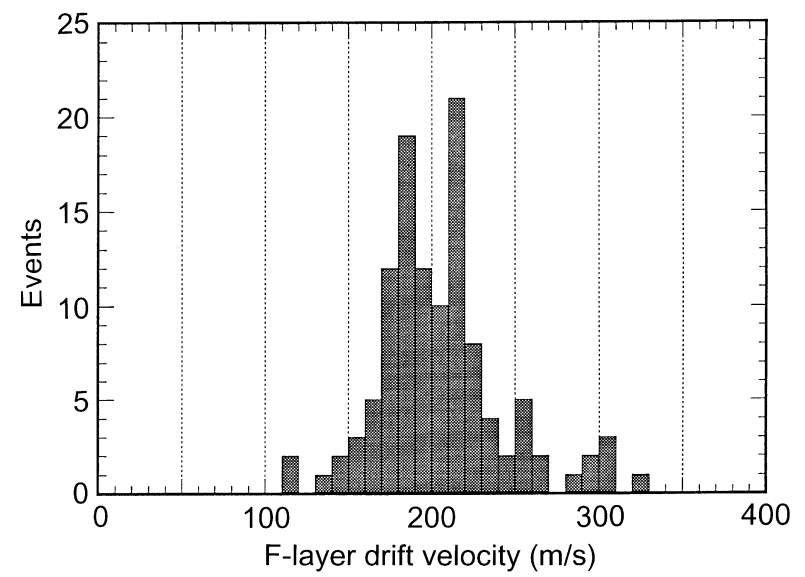

Fig. 16. F-layer drift-velocity histogram of the faster 116 retrograde events seen in the southern sky, and on lines of sight sensitive to motion in the zonal direction

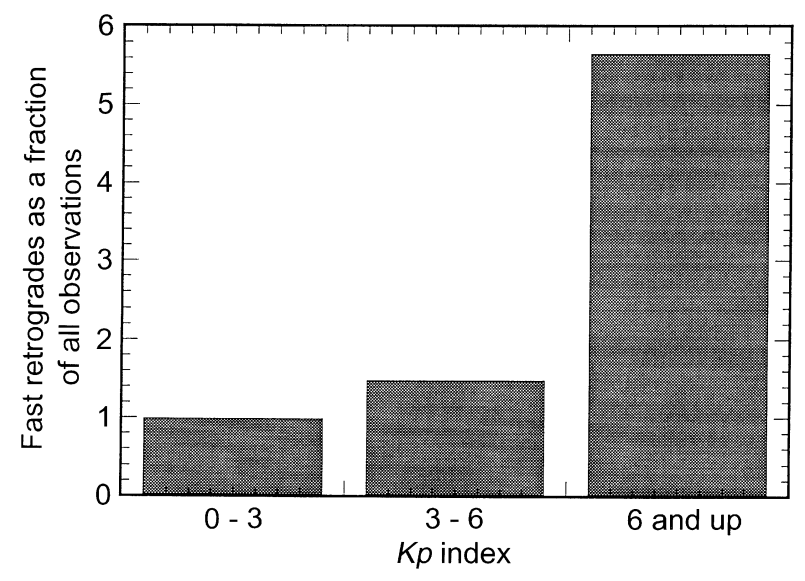

Fig. 17. Histogram of the $K_{p}$ index for the 116 fastest retrograde events shown in Fig. 16

which the disturbances were placed along the line of sight depends on accurately placing the measured trace azimuth perpendicular to the shadow of the magnetic field. This step is very sensitive to the geomagnetic-field model employed. We assume a simple tilted dipole, which for conditions of extreme geomagnetic activity could introduce errors in both magnitude and direction of the field. This, coupled with the intrinsic width of our measured azimuth distribution of $\pm 10^{\circ}$ (Fig. 7), can cause misidentifications of the disturbances' location along the line of sight, and thus cause all calculations of drift velocity to be inaccurate. We have, however, minimized these shortcomings to the best of our ability by not using events too low in altitude, thereby reducing the error introduced by the dipole approximation, and using only those events well described by a plane wave model and whose lines of sight possessed great differences in $\boldsymbol{B}$ shadow.

Finally, we wish to point out that the plane-wave model we employ explicitly assumes only one wave propagating across the array at a time. Although JE93 mentioned this fault, it is worth noting here that this does not allow for the detection of simultaneous multiple events. Therefore, it is quite likely that the $7 \%$ hit-rate quoted earlier severely underestimates the actual occurrence of this plasmaspheric phenomenon.

\section{Conclusions}

We have shown that through the reinterpretation of MED events using the $\boldsymbol{B}$-shadow technique, a much better explanation (compared to JE93) of their azimuths is found. MED events are now better identified and separated from the background of non-MED events. Furthermore, for appropriate lines of sight, MEDs can be accurately located in the plasmasphere independently of measured velocity. This results in (1) an $L$-shell distribution of smaller values than JE93, (2) a measurement of in situ plasma convection not previously possible, (3) measurements of a predominantly westward-convecting plasmasphere from $L=1.6$ to 3.0 , (4) 
westward drift speeds significantly higher than the eastward drift speeds (Fig. 15), and (5) the identification of "retrograde" events as in situ westward-convecting disturbances whose angular velocity exceeds the angular velocity of the VLA's westward-slipping line of sight. Finally, we realize westward convection in the inner plasmasphere is well-known, although the ability of a radio interferometer, such as the VLA, to detect it is not.

The next step in the study of plasmasphere convection using very long baseline radio interferometry is already underway. In recent work (Jacobson et al., 1995, 1996), an array of four (expanded to nine in the second work) antennas positioned in northern New Mexico utilizing radio beacons from geostationary satellites has observed a population of predominantly westward-moving, highvelocity, plane-wave disturbances in the plasmasphere. These geocorotating lines of sight used by the beacon array cannot observe corotating structures. Therefore the satellite events were not identified as MED events since, by definition, the old idea of MED implied corotating, not westward-moving events. However, we now recognize that the westward events observed by the satellite beacon array and the westward-convecting events seen by the VLA (Fig. 13) are the same.

The only real difference in the events detected by the VLA and the Los Alamos Beacon Array is the trace velocity. The VLA imparts large eastward velocities to the MED structures through the westward slipping of the line of sight. This line of sight motion Doppler-shifts the slower moving events up into ionospherically quiet frequencies, resulting in the detection of comparatively weaker convecting events. On the other hand, the beacon array only observes very fast convecting disturbances, thereby biasing the detected event pool towards highly disturbed, westward-convecting events. However, these biases do not affect the conclusion that we are able to detect plasmasphere convection, and therefore the future of the study of plasmasphere convection shall be continued with the cheaper, locally operated satellite beacon array.

It is our desire to perfect this technique and eventually monitor plasmasphere convection, and consequently, plasmasphere electric fields. The ability to produce quasi-real-time measurements of plasmasphere electric fields, at nearly insignificant cost, would be invaluable to the space weather forecasting effort.

Acknowledgements. The VLA is run by the National Radio Astronomy Observatory (NRAO) with funding from the National Science Foundation. We are grateful to the NRAO staff and user community for all the help provided during our use of the VLA. This research was performed under the auspices of the United States Department of Energy.

Topical Editor K.-H. Glassmeier thanks B. Fraser and J. P. Cerisier for their help in evaluating this paper.

\section{References}

Balmforth, H. F., R. J. Moffett, A. F. Smith, and G. J. Bailey, The effect of disturbed-time electric fields on the inner plasmasphere, Ann. Geophysicae, 12, 296-303, 1994.

Carlos, R. C., and R.S. Massey, The Los Alamos beacon receiver array, IEEE Trans. Geosci. Remote Sens., 32, 954-958, 1994.

Chappell, C. R., Detached plasma regions in the magnetosphere, J. Geophys. Res., 79, 1861-1869, 1974.

Fejer, B. G, D. T. Farley, C. A. Gonzalez, R. F. Woodman, and C. Calderon, F-region east-west drifts at Jicamarca, J. Geophys. Res., 86, 215-218, 1981.

Horwitz, J. L., R. H. Comfort, and C. R. Chappell, A statistical characterization of plasmasphere density structure and boundary locations, J. Geophys. Res., 95, 7937-7947, 1990.

Jacobson, A. R., and W. C. Erickson, A method for characterizing transient ionospheric disturbances using a large radiotelescope array, Astron. Astrophys., 257, 401-409, 1992a.

Jacobson, A. R., and W. C. Erickson, Wavenumber-resolved observations of ionospheric waves using the Very Large Array radio telescope, Planet. Space Sci., 40, 447-455, 1992 b.

Jacobson, A. R., and W. C. Erickson, Observations of electron density irregularities in the plasmasphere using the VLA radiointerferometer, Ann. Geophys, 11, 869-888, 1993.

Jacobson, A. R., R. C. Carlos, R. S. Massey, G. Wu, and G. W. Hoogeveen, Total-electron-content signatures of plasmaspheric motions, Geophys. Res. Lett., 22(18), 2461-2464, 1995.

Jacobson, A. R., G. W. Hoogeveen, R. C. Carlos, G. Wu, B. G. Fejer, and M. C. Kelley, Observations of inner-plasmasphere irregularities with a satellite-beacon radio-interferometer array, J. Geophys. Res., 101(A9), 19665-19682, 1996.

Kudeki, E., B. G. Fejer, D.T. Farley, and H. M. Ierkic, Interferometer studies of equatorial F-region irregularities and drifts, Geophys. Res. Lett., 8, 377-380, 1981.

Mazaudier, C., and S. V. Venkateswaran, Delayed ionospheric effects of the geomagnetic storms of March 22, 1979 studied by the sixth coordinated data analysis workshop (CDAW-6), Ann. Geophysicae., 8, 511-518, 1990.

Mcllwain, C. E., Coordinates for mapping the distribution of magnetically trapped particles, J. Geophys. Res., 66, 3681-3691, 1961.

Saxton, J. M., and A. J. Smith, Electric fields at $\mathrm{L}=2.5$ during geomagnetically disturbed conditions, Planet. Space Sci., 39, 1305-1320, 1991

Thompson, A. R., B. G. Clark, C. M. Wade, and P. J. Napier, The Very Large Array, Astrophys. J. Suppl. Ser., 44, 151-167, 1980 . 